\title{
Low Calcium Level
}

National Cancer Institute

\section{Source}

National Cancer Institute. Low Calcium Level. NCI Thesaurus. Code C162104.

A lower than average level of calcium in a sample. 